\subsection{MYCOPLASMA GENITALIUM IS AS FREQUENT A CAUSE OF URETHRITIS AS CHLAMYDIA TRACHOMATIS, AND HAS HIGH RATES OF GENOTYPIC RESISTANCE TO MACROLIDE ANTIBIOTICS}

doi:10.1136/sextrans-2013-051184.0192

M J Pond, A Nori, A A Witney, R Lopeman, P D Butcher, S T Sadiq. St George's, University of London, London, UK

Background When choosing empirical anti-microbial therapy for non-gonococcal urethritis (NGU) and cervicitis, efficacy against Chlamydia trachomatis over Mycoplasma genitalium is prioritised. However, M.genitalium is associated with reproductive sequelae in women and first-line recommended therapy with macrolide antibiotics differs to that for Chlamydia infection. We determined: M. genitalium and C.trachomatis frequency among symptomatic men with and without urethritis; prevalence of macrolide and fluoroquinolone associated genotypic resistance in M.genitalium; and the phylogenetic spread of genotypic M.genitalium antimicrobial resistance using a validated dual-locus typing system.

Methods Urethritis was diagnosed by combining urethral smear and clinical criteria. Nucleic acid amplification was used for detecting M.genitalium and C.trachomatis. Single nucleotide polymorphisms (SNPs) in the $23 \mathrm{~S}$ ribosomal RNA gene (23S rRNA), in gyrA, gyrB, and parC were detected by DNA sequencing. MG191 SNP typing and MG309 variable number tandem analysis were utilised to assess M.genitalium strain diversity.

Results 217 men were recruited. C.trachomatis and M.genitalium prevalence was $14.7 \%$ (95\% CI: 7.8-21.6) and 16.7\% (95\% CI: 9.524.0) respectively in NGU cases and both significantly higher than in those with no urethritis. $9 / 22$ (41\%; 95\% CI: 20\%-62\%) of M.genitalium strains had markers of macrolide associated genotypic resistance. Of $15 \mathrm{M}$.genitalium strains analysed only one possessed a parC mutation, associated with fluoroquinolone resistance. Duallocus typing assigned all M.genitalium strains to two major clusters, both of which contained diverse strains carrying resistant mutations. All strains were phylogenetically dispersed among international reference controls, typed using MG191.

Conclusion Frequency of M.genitalium was as high as C.trachomatis in NGU patients. More than $40 \%$ of M.genitalium strains had SNPs associated with macrolide resistance but fluoroquinolone associated genotypic resistance was rare. All strains were phylogenetically dispersed indicating that these infections were probably not part of a local clonal expansion. Treatment guidelines for NGU require re-appraisal in light of these findings.

\subsection{ESTIMATE OF THE PREVALENCE OF TRANSMITTED DRUG RESISTANCE (TDR) AND ACQUIRED DRUG RESISTANCE (ADR) IN A HIV RESISTANCE STUDY OF THE GERMAN CLINSURV-HIV COHORT}

doi:10.1136/sextrans-2013-051184.0193

'B Bartmeyer, 'D Schmidt, 'C Kollan, ${ }^{2} \mathrm{G}$ Fätkenheuer, ${ }^{3} \mathrm{H}$ Stellbrink, ${ }^{4} \mathrm{~J}$ Bogner, ${ }^{5} \mathrm{~B} O$ Jensen, ${ }^{6} \mathrm{M}$ Stoll, ${ }^{1} \mathrm{C}$ Kücherer, ${ }^{1} 0$ Hamouda. ${ }^{1}$ Robert Koch Institute, Berlin, Germany; ${ }^{2}$ Clinic for Internal Medicine, University of Cologne, Germany; ${ }^{3} \mathrm{CH}$ Study Centre Hamburg, Hamburg, Germany; ${ }^{4}$ Dept. of Infectious Diseases, Medical Clinic, Ludwig Maximilians University Munich, Germany; ${ }^{5}$ Clinic of Gastroenterology and Hepatology, Heinrich Heine University Düsseldorf, Germany; ${ }^{6}$ Clinic for Immunology and Rheumatology, Medical University Hanover, Germany

Aim To estimate the prevalence of HIV-TDR and ADR in one resistance study of the German ClinSurv-HIV cohort.

Method The ClinSurv study is a national open multi-centre long term observational cohort with 15 participating clinical centres ( $n=16,750$ patients; 31.12.2011). In a resistance study all ClinSurv patients in five centres were identified. Sequences were processed through the Stanford University Genotypic Resistance Interpretation
Algorithm (www. hivdb.stanford.edu; HIVdb version 6.1.1F; 2012 webService version beta-1.0.1) to identify mutations and to determine drug susceptibility. Sequences were analysed by using different lists of mutations (Bennett D. 2009; Johnson V. 2011). Trends in the prevalence of drug resistance mutations were calculated by logistic regression.

Results A total of 9,528 patients from five study centres were included into analysis. 4,989 viral sequences were collected from $34 \%(3,267 / 9,528)$ of these patients. $47 \%(2,365 / 4,989)$ of sequences were produced from patients being treatment naïve and $50 \%$ $(2,495 / 4,989)$ from patients under treatment. TDR was identified in $10 \%(203 / 1,950)$ of viral strains. The prevalence of TDR over time was stable at $10.4 \%$ (95\% CI 9.1-11.8; OR: 0.98; 95\% CI 0.92-1.04; $\left.\mathrm{p}_{\text {for trend }}=0.6 ; 2001-2011\right)$. NRTI-resistance was determined in 7\% (128/1,950), followed by 3\% NNRTI- and PI-resistance, respectively (NNRTI: 61/1,950; PI: 56/1,950). Prevalence of ADR in treated patients was high (61\%; 1,500/2,453 of sequences) but declined significantly over time (OR: $0.8 ; 95 \%$ CI $0.77-0.83$; $\mathrm{p}_{\text {for trend }}<0.001$; 2001-2011). Within drug classes NNRTI-resistance was predominant (56\%; 834/1503), followed by NRTI-resistance in $52 \%$ $(1,139 / 2,194)$ of sequences of patients with $\mathrm{ADR}$ exposed to these drug classes. PI-resistance was identified in 30\% (543/1778). Integrase-resistance was determined in $8 \%$ (13/161) of integrasesequences.

Discussion Prevalence of TDR is highly stable in this unselected study population, whereas $\mathrm{ADR}$ declined significantly over the time, indicating that this decline was presumably influenced by ART related effects, broader resistance testing and resistance test guided therapy.

\subsection{ACCEPTABILITY OF HPV VACCINATION AMONG PARENTS OF ADOLESCENT SCHOOL GOING GIRLS IN MYSORE CITY, INDIA}

doi:10.1136/sextrans-2013-051184.0194

${ }^{1,2} \mathbf{K}$ Krupp, 'V Srinivas, ${ }^{3} \mathrm{~L}$ Marlow, ${ }^{2} \mathrm{~T}$ Li, ${ }^{2} \mathrm{~A}$ Albetini, ${ }^{1} \mathrm{~S}$ Gowda, ${ }^{2} \mathrm{~A}$ Arun, ${ }^{1 P}$ Jaykrishna, 2.1P Madhivanan. ${ }^{1}$ Public Health Research Institute of India, Mysore, India; ${ }^{2}$ Robert Stempel College of Public Health \& Social Work, Florida International University, Miami, FL, United States, ${ }^{3}$ Health Behavior Research Centre, Department of Epidemiology and Public Health, UCL, London, UK

Background One in every four reported cases of cervical cancer occurs in India. While mortality from the disease has all but disappeared in industrial countries, 74,000 Indian women still die each year from this preventable cancer. It has been estimated that widespread uptake of HPV vaccine by adolescent girls could reduce this high incidence and mortality by approximately two-thirds. This study explores correlates of HPV vaccine acceptability among parents of adolescent school-going girls in urban Mysore, India.

Methods Between August and December of 2011, participants were selected by stratified, multi-stage random sampling in schools located in Urban Mysore. Questionnaires were sent home with a random sample of 800 adolescent girls 11-15 years of age attending 10 schools in Mysore city to be completed by a parent. Logistic regression was used to assess factors associated with parental acceptability of HPV vaccine.

Results 797 completed surveys (99.6\%) were received back from parents. About $72 \%$ of respondents would accept the HPV vaccine for their daughters. Vaccine acceptance was higher among participants who had experienced cancer in their family (OR: 1.69, 95\% CI: 1.07, 2.65), or perceived that their family doctor (5.04; CI 3.27, 7.76 ) or spouse (5.01; CI: $3.20,7.87)$ would approve. Parents having concerns about vaccinations in general (0.38; CI: $0.25,0.57)$, vaccine side-effects (0.65; CI: $0.45,0.94)$, vaccine safety (0.64; CI: $0.42,0.97)$ or the possibility that their daughter might become sexually active (0.71; CI: $0.28,0.76)$ had lower odds of accepting HPV vaccination. 
Parents belonging to the Muslim religion (0.54; CI: 0.37, 0.80) had lower odds of vaccine of HPV vaccine acceptance.

Conclusion The majority of parents of school-going adolescent girls in Mysore found HPV immunisation acceptable. Further research is needed to understand the issues associated with HPV vaccination in different religious groups in India.

\subsection{ASSESSING THE EFFECTIVENESS OF THE HUMAN PAPILLOMAVIRUS (HPV) VACCINATION PROGRAMME IN VICTORIA, AUSTRALIA}

doi:10.1136/sextrans-2013-051184.0195

${ }^{1} S$ M Garland, ${ }^{2} \mathrm{~S} 0$ 0sborne, ${ }^{3} \mathrm{E} J$ Young, ${ }^{4} \mathrm{~J} \mathrm{M}$ L Brotherton, ${ }^{3} \mathrm{~S} N$ Tabrizi, ${ }^{4} \mathrm{D}$ M Gertig, ${ }^{3} Y$ Jayasinghe, on behalf of the VACCINE study group. 'The Royal Women's Hospital, Murdoch Childrens Research Institute, Parkville, Australia; ${ }^{2}$ The Royal Women's Hospital, Murdoch Childrens Research Institute, University of Melbourne, Parkville, Australia; ${ }^{3}$ Murdoch Childrens Research Institute, Parkville, Australia; ${ }^{4}$ University of Melbourne, Victorian Cytology Service, Parkville, Australia

Background Quadrivalent HPV vaccination has been available in Australia through the National HPV Vaccination Program since 2007. The VACCINE study aims to evaluate the effectiveness of the programme by assessing the prevalence of vaccine targeted HPV genotypes (HPV 6, 11, 16 and 18). We aim to detect any decrease in prevalence of vaccine-targeted HPV genotypes amongst young women in vaccine eligible cohorts and to independently measure vaccine coverage in young Victorian women.

Methods Young Victorian women aged 18-25 are recruited using the social networking site Facebook. Participants complete an online questionnaire and those sexually active are asked to provide a selfcollected vaginal swab. Swabs are genotyped using a Linear Array HPV genotyping test (Roche Diagnostics).

Results To date, 623 (of 1570) females have been recruited into the study and 477 participants have completed the study. 71\% (440) were sexually active and of these women $373 / 440$ (85\%) provided a self-collected swab, of which $75 \%$ were negative for HPV. Of the 95 cases positive for HPV, only 6 cases of HPV 16 have been recorded, and no cases of HPV 6, 11 or 18 have been identified. The prevalence of HPV16 $(1.6 \%)$ is significantly lower than that detected from prevaccine age matched Victorian women cervical specimens $(9.4 \%)$ $\left(\chi_{(1)}^{2}=18.3, p<0.001\right)$. Based on self-reported vaccination status, $85 \%$ of women aged $18-21$ years old in our sample have received at least one dose of the HPV vaccine, compared with $83 \%$ for women aged 22-25. Cross protection against HPV 31 is also being seen $\left(0.5 \%\right.$ versus $\left.5.6 \%, \chi_{(1)}^{2}=14.2, p<0.001\right)$.

Conclusions Preliminary data from the VACCINE study suggest a significant decline in the prevalence of vaccine-targeted HPV genotypes. These results support the hypothesis that the HPV vaccine programme is effective in reducing HPV genotypes 6, 11, 16 and 18 amongst populations offered the vaccine.

\subsection{USE OF SURFACTANT VESICLES AS A POTENTIAL GONOCOCCAL VACCINE DELIVERY SYSTEM TO GENERATE ANTIBODY AGAINST NEISSERIAL LIPOOLIGOSACCHARIDE}

doi:10.1136/sextrans-2013-051184.0196

'D Stein, 'L Zimmerman, ${ }^{2} \mathrm{~J}$ Park, ${ }^{2} \mathrm{~L}$ Stocker, ${ }^{2} \mathrm{P}$ DeShong. 'University of Maryland, Department of Cell Biology and Molecular Genetics, College Park, MD, United States; 'University of Maryland, Department of Chemistry and Biochemistry, College Park, MD, United States

Background To date, no one has been able to exploit the immunological potential of neisserial lipooligosaccharide (LOS) as a vaccine candidate.

Methods We have developed a glycoconjugate vaccine (TRIAD) that contains native LOS derived from Neisseria gonorrhoeae F62)
$\Delta \operatorname{lgtD}$ (a strain that produces lacto-N-neotetraose LOS and a peptide (PADRE) that possesses the ability to bind to a large number of HLA class II molecules, inserted into a surfactant vesicle. TRIAD is a catanionic surfactant vesicle formulation and the resulting vesicle is stable at room temperature for years, unlike a typical liposome. TRIAD is so robust that it can be autoclaved without any appreciable loss of structural integrity.

Results Using TRIAD that contained LOS and PADRE at a ratio of 10:1, and immunising with $2 \mu \mathrm{g}$ of LOS equivalent, we were able to demonstrate that our vaccine induces a high titer anti-LOS antibody response, with the majority of the elicited antibody being IgG. Intraperitoneal immunisation of mice with our vaccine construct produced no observable adverse effects in mice, while intraperitoneal immunisation with equivalent amounts of purified LOS induced significant adverse effects.

Conclusions Our surfactant vesicle platform possesses all of the advantages seen with traditional liposome formulations, without any of the inherent problems associated with liposome-mediated vaccines. This vaccine platform readily lends itself to further modifications in that it is possible to include additional neisserial proteins into the vaccine via a novel whole cell extraction protocol. We believe that this will allow us to generate a universal vaccine able to protect against all serotypes of $N$. meningitidis.

\subsection{0 - Sexual partnerships and networks}

\section{O20.1 TWO-MODE ANALYSIS OF HIV, HCV AND RISK BEHAVIOURS: A PLACE-BASED ANALYSIS OF MOST AT- RISK POPULATIONS IN WINNIPEG, CANADA}

doi:10.1136/sextrans-2013-051184.0197

'S Y Shaw, 'C Green, ${ }^{2} \mathrm{~J}$ L Wylie. 'University of Manitoba, Winnipeg, MB, Canada, ${ }^{2}$ Cadham Provincial Laboratory, Winnipeg, MB, Canada

Background The HIV epidemic has shown a considerable amount of heterogeneity, complicating the design and placement of prevention, intervention and treatment programmes. Place-based analyses providing specific information on pathogen prevalence, risk behaviours and other micro-level behaviours can help to target public health responses.

Methods Data were from a cross-sectional survey of most-at-risk populations (MARPs) from Winnipeg, Canada. Respondents were recruited through respondent-driven sampling, and biological, behavioural and egocentric network data were collected. Respondents named locations where they frequented and where they engaged in risk behaviours, including the use of crack cocaine, injectable and non-injectable drug use, and solvents; and either seeking sex work clients or sex workers. Locations were geo-coded up to Statistics Canada dissemination areas. Two-mode network visualisation and centrality, degree, and betweenness measures were generated using UciNet (V.6).

Results From a sample size of 600 , nine locations were named by 10 or more respondents. The following results pertain only to these nine locations (N: 231). Locations corresponded to three "hot spots" in Winnipeg's inner and outer core areas. Across the sample, HIV and HCV prevalence was $9.8 \%$ and $51.5 \%$, respectively. Prevalence varied considerably by location, ranging from $0 \%$ to $15 \%$ for HIV and $20 \%$ to $70 \%$ for HCV. Degree ranged from 0.054 to 0.330 , closeness from 0.178 to 0.411 and betweenness from 0.054 to 0.521 . No association between prevalence of HIV and HCV and network metrics was found. Substantial heterogeneity in pathogen prevalence and risk behaviour was observed by location, while pathogen, risk and mixing characteristics of populations bridging the nine locations were made apparent by two-mode visualisation.

Conclusion Two-mode analysis of egocentric network data revealed geographic clustering of risk behaviours, while at the same 